\title{
Excitation and Stabilization of Passive Dynamics in Locomotion using Hierarchical Operational Space Control*
}

\author{
Marco Hutter, Christian Gehring, Michael Bloesch, Mark Hoepflinger, Péter Fankhauser, Roland Siegwart ${ }^{1}$
}

\begin{abstract}
This paper describes a hierarchical operational space control (OSC) method based on least square optimization and outlines different ways to reduce the dimensionality of the optimization vector. The framework allows to emulate various behaviors by prioritized task-space motion, joint torque, and contact force optimization. Moreover, a methodology is introduced to partially excite the natural dynamics of the robot by open-loop motor regulation while the entire behavior is stabilized by hierarchical OSC. As a major contribution, the presented control strategies are tested and validated in real hardware walking, trotting, and pronking experiments using a fully torque controllable quadrupedal robot.
\end{abstract}

\section{INTRODUCTION}

Recent advances in design and actuation of legged robotic systems make sophisticated, torque controllable robots available to the research community. In contrast to traditionally stiff and position controllable devices, these new systems can softly (and hence safely) interact with their environment [1]. This fundamental change opens wide opportunities to bring novel control concepts from simulations into real world applications. Instead of slow, static walking based on kinematic motion planning and execution [2], these new principles have the potential to enable agile, highly dynamic, and versatile maneuvers. Torque controllability allows to create sophisticated behaviors by simultaneously emulating and optimizing desired task-space dynamics, joint torques, or contact forces.

A particularly interesting approach to control complex, torque controllable robotic systems is hierarchical operational space control (OSC). Thereby, the desired system dynamics are described in task space at different points of interest on the robot [3]. To give some examples, this can be controlling the center of gravity $(\mathrm{CoG})$ to ensure stability, performing stepping tasks with feet, or keeping a certain optimal posture. The robot behavior is completed by force and torque objectives like applying a desired contact force, improving efficiency, or avoiding slippage. Such prioritized control formulations were first proposed in [4] in the context of inverse kinematics control and were later generalized for an arbitrary number of tasks [5]. Along the lines of the seminal work on OSC by Khatib [6], his group extended the task prioritization ideas to hierarchical OSC for floating-base systems. They set up a framework to solve task-space inverse

\footnotetext{
*This work was supported in part by the Swiss National Science Foundation through the National Centre of Competence in Research Robotics, as well as by the Hans Eggenberger Foundation

${ }^{1}$ M. Hutter, C. Gehring, M. Bloesch, M. Hoepflinger, P. Fankhauser, R. Siegwart are with the Autonomous Systems Lab, ETH Zurich, Switzerland mahutter@ethz.ch
}

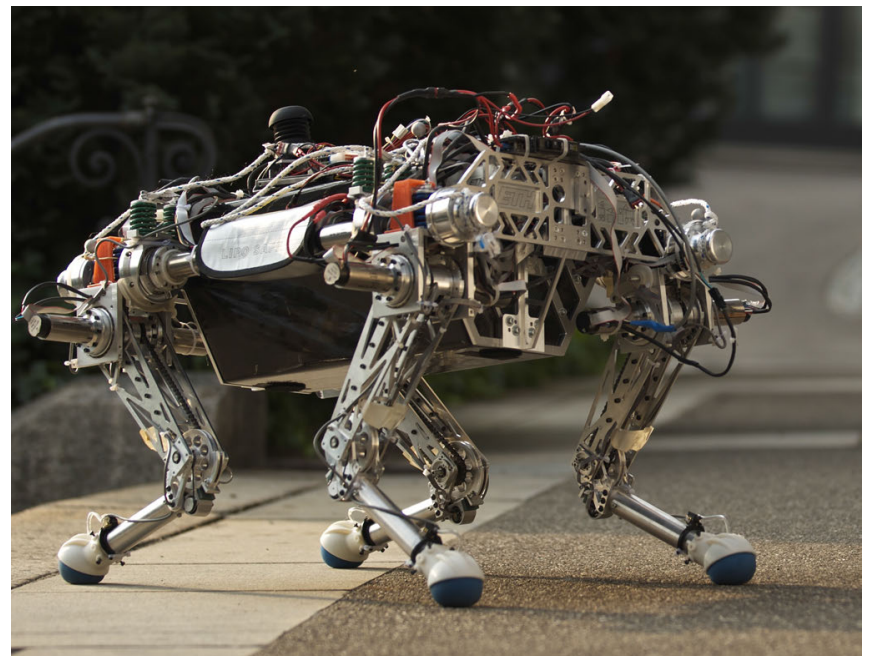

Fig. 1. StarlETH is a medium-dog-sized quadrupedal robot driven by precisely torque controllable series elastic actuators [17].

dynamics with various priorities for both motion as well as force/torque tasks [7] and recently released this powerful software package for public [8]. Similar methods were also published in [9] based on the formulation of a minimization problem. All these hierarchical control methods guarantee by iterative null-space projection that a task never impairs a higher prioritized task. Other methodologies (e.g. [10]) understand the hierarchy of tasks as a set of constrained quadratic programs (QP) and use numerical solvers [11] to fast and robustly resolve the redundancy as it can occur in complex robotic systems. Similar to the iterative nullspace projection, dedicated QP formulations [12] can also be applied for inverse dynamics methods, e.g. [13], [14]. Moreover, inequality tasks [15] as required to describe position, torque, or force limitations, can be very naturally integrated using slack variables. This was shown in [16] for kinematic problems and [14] later suggested to augment this method for inverse dynamics.

Hierarchical OSC or inverse dynamics methods in general are extremely beneficial for stabilizing a certain motion or behavior. However, finding the appropriate target behavior tends to be a complex problem that requires substantial planning and/or optimization [18]. On top of this, the performance is limited as the actuators are used in a pure feedback manner with an additional torque control loop on joint level that tends to actively suppress passive system dynamics. This is particularly true for robots like StarlETH (Fig. 1, [17]), which is based on highly compliant and precisely torque controllable series elastic actuators (SEA) [19] in all 
joints. This actuation principle uses springs to decouple the motor from the joint and hence has the advantage that it can increase the peak power and actuator efficiency if properly excited [20]. In fact, such passive dynamic mechanisms are not only exploited by several legged robots (e.g. [21], [22]), but are also one of the main reasons of the high energetic efficiency in human and animal locomotion [23].

The theoretical contribution of this paper is twofold. First, we present a way of prioritized OSC based on least square (LS) optimization with a reduced optimization vector which (i) is generic, (ii) simple and clearly understandable, and (iii) efficient to implement and solve. Second, we outline a simple methodology that allows to operate certain motors of the robot in a open-loop manner to excite the passive system dynamics, while the remainder of the actuators is used to stabilize the motion based on hierarchical OSC. In contrast to many other contributions in this field, the presented algorithms are not only tested in simulations but additionally validated on the quadrupedal robot StarlETH under real world conditions.

\section{HIERARCHICAL OSC}

\section{A. System Dynamics}

The system dynamics in terms of equations of motion (EoM) of a floating-base system are described in the form

$$
\mathbf{M} \ddot{\mathbf{q}}+\mathbf{h}+\mathbf{J}_{s}^{T} \mathbf{F}_{s}=\mathbf{S}^{T} \boldsymbol{\tau}
$$

with the mass matrix $\mathbf{M}(\mathbf{q})$, the force vector $\mathbf{h}(\mathbf{q}, \dot{\mathbf{q}})$ as the sum of Coriolis, centrifugal and gravitational forces, the ground contact forces $\mathbf{F}_{s}$, the corresponding Jacobian $\mathbf{J}_{s}(\mathbf{q})$, and the actuator torque $\boldsymbol{\tau}$. Everything is expressed as a function of the $n$ generalized coordinates $\mathbf{q}=\left(\mathbf{q}_{b} ; \mathbf{q}_{r}\right)$, whereby the selection matrix $\mathbf{S}=\left[\begin{array}{ll}\mathbf{0}_{n_{r} \times n_{b}} & \mathbf{I}_{n_{r}}\end{array}\right]$ separates the $n_{r}=n-n_{b}$ actuated joint coordinates $\mathbf{q}_{r}$ from the $n_{b}=6$ unactuated floating-base coordinates $\mathbf{q}_{b}$. Floating base systems interact with the environment at continuously changing contact points. Often, and in particular for control, this interaction is modeled as a hard contact constraint

$$
\ddot{\mathbf{r}}_{s}=\mathbf{J}_{s} \ddot{\mathbf{q}}+\dot{\mathbf{J}}_{s} \dot{\mathbf{q}}=\mathbf{0},
$$

which implies that the points in contact with the environment are not supposed to move.

\section{B. Hierarchical OSC as LS Problem}

In a nutshell, hierarchical OSC of floating base systems, e.g. [24], can be understood as simultaneous execution of different tasks with different priorities in compliance with the system dynamics (1) and contact constraints (2). A generic and well comprehensible formulation is to describe this as a set of least squares problems

$$
\min _{\mathbf{x}}\|\mathbf{A x}-\mathbf{b}\|_{2}
$$

with the optimization variable $\mathrm{x}$ being a stacked vector of joint accelerations $\ddot{\mathbf{q}}$, joint torques $\tau$, and contact forces $\mathbf{F}_{s}$ :

$$
\mathbf{x}=\left(\begin{array}{c}
\ddot{\mathbf{q}} \\
\boldsymbol{\tau} \\
\mathbf{F}_{s}
\end{array}\right)
$$

1) Force Elimination: Instead of expressing the optimization as a function of the full optimization vector (4) like in [9], we can make use of tools that are common from inverse dynamics control. By using projector matrices $\mathbf{P}_{F}$ such as a dynamically consistent null-space projector [25], a QR decomposition of $\mathbf{J}_{s}$ [26], or Jacobian null-space projections [27], (1) reduces to

$$
\mathbf{P}_{F}(\mathbf{M} \ddot{\mathbf{q}}+\mathbf{h})=\mathbf{P}_{F} \mathbf{S}^{T} \boldsymbol{\tau}
$$

since $\mathbf{P}_{F} \mathbf{J}_{s}^{T}=\mathbf{0}$. As a benefit, this decreases the optimization dimensionality to $n+n_{r}$ :

$$
\mathbf{x}=\left(\begin{array}{l}
\ddot{\mathbf{q}} \\
\boldsymbol{\tau}
\end{array}\right) \text {. }
$$

As shown in [28], the choice of projector does not change the controller but only the way how redundancy is resolved.

2) Torque Elimination: An even simpler approach that is inspired by [29] is to make use of the structure of the actuation selection matrix $\mathbf{S}^{T}$. To this end, we consider only the unactuated part of the dynamics

$$
\mathbf{P}_{\tau}\left(\mathbf{M} \ddot{\mathbf{q}}+\mathbf{h}+\mathbf{J}_{s}^{T} \mathbf{F}_{s}\right)=\mathbf{0},
$$

with $\mathbf{P}_{\tau}=\left[\begin{array}{ll}\mathbf{I}_{n_{b}} & \mathbf{0}_{n_{b} \times n_{r}}\end{array}\right]$ and $\mathbf{P}_{\tau} \mathbf{S}^{T}=\mathbf{0}$. In fact, analogous to the null-space calculation of the inverse dynamics methods, $\mathbf{P}_{\tau}$ represents the null-space of $\mathbf{S}^{T}$ and reduces the optimization vector to

$$
\mathbf{x}=\left(\begin{array}{c}
\ddot{\mathbf{q}} \\
\mathbf{F}_{s}
\end{array}\right)
$$

Using this reduced set of optimization variables, the actuator torque is given by

$$
\boldsymbol{\tau}=\mathbf{S}\left(\mathbf{M} \ddot{\mathbf{q}}+\mathbf{h}+\mathbf{J}_{s}^{T} \mathbf{F}_{s}\right) .
$$

Without loss of generality, this method has the benefit that all projection matrices are known before runtime. In the follow, we will focus on this method.

\section{LS with Torque Reduced Optimization Vector}

The behavior of a robotic system is determined by a set of tasks that are simultaneously executed. With highest priority, the system dynamics (1) has to be fulfilled:

$$
\begin{gathered}
\min _{\mathbf{x}}\left\|\mathbf{P}_{\tau}\left(\mathbf{M} \ddot{\mathbf{q}}+\mathbf{h}+\mathbf{J}_{s}^{T} \mathbf{F}_{s}\right)\right\|_{2} \\
\mathbf{A}_{0}=\mathbf{P}_{\tau}\left[\begin{array}{ll}
\mathbf{M} & \mathbf{J}_{s}^{T}
\end{array}\right], \quad \mathbf{b}_{0}=-\mathbf{P}_{\tau} \mathbf{h} .
\end{gathered}
$$

With second priority, the contact condition (2) is ensured:

$$
\begin{gathered}
\min _{\mathbf{x}}\left\|\mathbf{J}_{s} \ddot{\mathbf{q}}+\dot{\mathbf{J}}_{s} \dot{\mathbf{q}}\right\|_{2} \\
\mathbf{A}_{1}=\left[\begin{array}{ll}
\mathbf{J}_{s} & \mathbf{0}
\end{array}\right], \quad \mathbf{b}_{1}=-\dot{\mathbf{J}}_{s} \dot{\mathbf{q}} .
\end{gathered}
$$

Given the structure of $\mathbf{A}_{0}$ and $\mathbf{A}_{1}$, it can be proven that both tasks are exactly fulfilled, i.e. that the least squares optimization results in zero costs. Furthermore, to create the desired task- or operational space motion $\ddot{\mathbf{r}}_{i \text {,des }}$, a set of motion optimization tasks can be added:

$$
\begin{gathered}
\min _{\mathbf{x}}\left\|\ddot{\mathbf{r}}_{i, \mathrm{des}}-\ddot{\mathbf{r}}_{i}\right\|_{2} \\
\mathbf{A}_{i}=\left[\begin{array}{ll}
\mathbf{J}_{i} & \mathbf{0}
\end{array}\right], \quad \mathbf{b}_{i}=\ddot{\mathbf{r}}_{i, \mathrm{des}}-\dot{\mathbf{J}}_{i} \dot{\mathbf{q}}
\end{gathered}
$$


Moreover, contact force optimization tasks are written as

$$
\begin{gathered}
\min _{\mathbf{x}}\left\|\mathbf{W}_{i, F}\left(\mathbf{F}_{i, \mathrm{des}}-\mathbf{F}_{i}\right)\right\|_{2} \\
\mathbf{A}_{i}=\left[\begin{array}{ll}
\mathbf{0} & \mathbf{W}_{i, F}
\end{array}\right], \quad \mathbf{b}_{i}=\mathbf{W}_{F, i} \mathbf{F}_{i, \mathrm{des}},
\end{gathered}
$$

with $\mathbf{W}_{i, F}$ being a selection matrix of the respective force directions and $\mathbf{F}_{i \text {,des }}$ the desired contact forces. As it was shown in [30], it is possible to integrate the local tangential planes in $\mathbf{W}_{F}$ which drastically reduces the risk of slippage in uneven ground. Finally, using (9), torque optimization tasks are stated as

$$
\begin{gathered}
\min _{\mathbf{x}}\left\|\mathbf{W}_{i, \tau}\left(\boldsymbol{\tau}_{i, \mathrm{des}}-\boldsymbol{\tau}_{i}\right)\right\|_{2} \\
\mathbf{A}_{i}=\mathbf{W}_{i, \tau} \mathbf{S}\left[\begin{array}{ll}
\mathbf{M} & \mathbf{J}_{s}^{T}
\end{array}\right], \mathbf{b}_{i}=\mathbf{W}_{i, \tau}\left(\boldsymbol{\tau}_{i, \mathrm{des}}-\mathbf{S h}\right),(1,
\end{gathered}
$$

with $\mathbf{W}_{i, \tau}$ being the torque weighting and selection matrix and $\boldsymbol{\tau}_{i, \text { des }}$ the desired torque vector for the corresponding directions.

\section{Solving a Prioritized LS Problem}

A set of least squares problems in the form of (3) with different priorities can be solved by iterative null-space projection. As already illustrated by [5] in a similar manner, the requirement that a task is not allowed to influence any task with higher priority can be formulated by defining $\mathbf{x}$ as a sum of task specific $\mathbf{x}_{i}$ pre-multiplied with the null-space projection matrix $\mathbf{N}_{i}$ of higher prioritized tasks:

$$
\mathbf{x}=\sum_{k=1}^{n_{T}} \mathbf{N}_{k} \mathbf{x}_{k} .
$$

The null-space projector $\mathbf{N}_{i}$ is defined as $\mathbf{N}_{i}=$ $\mathcal{N}\left(\left[\begin{array}{lll}\mathbf{A}_{1}^{T} & \ldots & \mathbf{A}_{i-1}^{T}\end{array}\right]^{T}\right)$ with $\mathbf{N}_{1}=\mathbf{I}$ and the sufficient property

$$
\mathbf{A}_{i} \mathbf{N}_{j}=\mathbf{0} \quad \forall i<j .
$$

It can be computed using the pseudo inverse as outlined in e.g. [5], [25] or by a singular value decomposition (SVD) [31] which shows better numerical stability. Using property (16), the prioritized minimization problem (3) can be solved for each task individually by inserting (15) and solving for $\mathbf{x}_{i}$ :

$$
\begin{aligned}
\mathbf{A}_{i} \mathbf{x}-\mathbf{b}_{i} & =\mathbf{A}_{i} \sum_{k=1}^{n_{T}} \mathbf{N}_{k} \mathbf{x}_{k}-\mathbf{b}_{i} \\
\mathbf{x}_{i} & =\left(\mathbf{A}_{i} \mathbf{N}_{i}\right)^{+}\left(\mathbf{b}_{i}-\mathbf{A}_{i} \sum_{k=1}^{i-1} \mathbf{N}_{k} \mathbf{x}_{k}\right) .
\end{aligned}
$$

It can be analytically proven that (i) tasks with higher priority are not influenced by tasks with lower priority and (ii) that the solution is globally optimal. For the corresponding proofs, the interested reader is referred to [31]. Furthermore, as long as the rows of $\mathbf{A}_{i} \mathbf{N}_{i}$ are linearly independent, the task is exactly fulfilled $\left(\mathbf{A}_{i} \mathbf{x}=\mathbf{b}_{i}\right)$. To ensure numerical robustness also in case $\mathbf{A}_{i} \mathbf{N}_{i}$ being rank deficient, we use a SVD pseudo inversion [32].

\section{E. Inequality Constraints}

To describe the behavior of complex legged robots, it is often required to additionally integrate inequality constraints

$$
\mathbf{A}_{i} \mathbf{x} \leq \mathbf{b}_{i}
$$

in the proposed control framework, e.g. to handle joint angle limitation or motor torque saturation on task-space level [15], [13]. The simplest possibility is to activate the corresponding inequality task as equality tasks as soon as the inequality constraint is violated. By assigning a higher priority than to all other tasks, we can ensure that (in case there is a feasible solution to the problem) the actual solution fulfills the inequality constraint. However, to handle multiple independent inequality tasks as well as to activate and deactivate them, it is required to always iterate through all possible combinations of active and inactive constraints, which can become computationally intensive. While this approach works well for our application with a rather low number of inequality constraints, a more elaborated and natural method was presented in [16]. Using a constrained quadratic programming $(\mathrm{QP})$ formulation, they propose to integrate the inequality tasks with slack variables and to solve the problem using numerical QP solvers.

\section{ExPloiting PASSIVE DyNAMiCS}

As outlined in the introduction, it can be required that certain actuators of the robot, indicated by the selection matrix $\mathbf{S}_{P D}$, are operated in an open-loop manner to optimally excite the passive system dynamics

$$
\boldsymbol{\tau}_{P D}=\mathbf{S}_{P D} \mathbf{S} \boldsymbol{\tau}
$$

while the remainder of the actuators is dedicated to stabilize the target behavior based on the outlined hierarchical OSC. Such combined control problem can be solved by considering the passive dynamics as measurable system disturbance $\boldsymbol{\tau}_{P D}=\boldsymbol{\tau}_{P D \text {, meas }}$, which is only possible since torque controllable robots like StarlETH feature precise joint torque sensing capabilities. In particular for SEAs, the natural joint spring dynamics during stance has typically a frequency clearly below $10 \mathrm{~Hz}$, which is significantly lower than the sampling respectively control frequency $(400 \mathrm{~Hz}$ for StarlETH). Hence, these torque measurements can be directly integrated as a single task of high priority (right after the system dynamics and contact constraints)

$$
\mathbf{A}_{2}=\mathbf{S}_{P D} \mathbf{S}\left[\begin{array}{ll}
\mathbf{M} & \mathbf{J}_{s}^{T}
\end{array}\right], \quad \mathbf{b}_{2}=\boldsymbol{\tau}_{P D, \text { meas }}-\mathbf{S}_{P D} \mathbf{S h},
$$

implying that the joint torque remains constant over two sampling steps.

\section{RESUltS}

In the first set of experiments, the applicability and robustness of the proposed prioritized LS optimization is demonstrated in static and dynamic gaits. In the second set, the exploitation of passive dynamics is evaluated in highly dynamic pronking maneuvers and compared to pure feedback control. All experiments are first tested in simulation and 


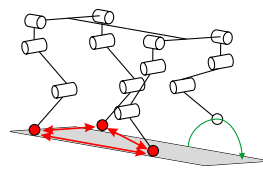

(a) Walk

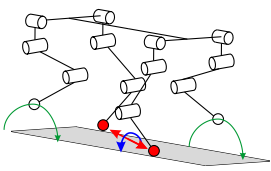

(b) Trot

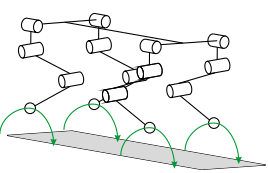

(c) Pronk
Fig. 2. The present control strategy was tested in three gaits.

subsequently validated in hardware experiments with the freely moving robot being operated on a treadmill using pure onboard intelligence (no external motion capture system). All kinematic and dynamic system properties are take from the CAD model and the OSC gains were tuned by hand.

\section{A. Static Walking}

For static gaits (Fig. 2(a)), the robot has at least three legs in simultaneous ground contact. This allows to fully define the motion of the main body. In addition to setting the priority of the system dynamics (1) and contact constraint (2) consistency to the highest level, regulation of the body position and orientation is chosen as less important tasks. The body position is shifted between the successive support triangles to maintain stability while walking and the body orientation is kept constant.

Since at least three legs are in simultaneous ground contact, the system has internal contact directions (red arrows in Fig. 2(a)) that allow to change the contact force or joint torque distribution without influencing the motion. Hence, as task with lowest priority we choose either to optimize for efficiency or for robustness against slippage. Since the energy consumption of electric motors is often quantified by the integral over current square [33], efficiency can be optimized by torque minimization $\left(\mathbf{W}_{\tau}=\mathbf{I}, \boldsymbol{\tau}_{\text {des }}=\mathbf{0}\right)$. (Risk of) slippage on the other side is mainly depending on the local tangential forces and can hence be lowered by a force minimization task that selects the local tangential forces by $\mathbf{W}_{F}$. For flat ground, $\mathbf{W}_{F}$ can be simply chosen as unitary matrix, which additionally evenly distributes the vertical load.

We conducted two walking sequences over four full gait cycles with either energy (Fig. 3, black) or risk of slippage minimization (Fig. 3, red). Between this two experiments, the average absolute difference of the main body position was only $3.6 \mathrm{~mm}$ and the body orientation in roll, pitch, and yaw diverged less than $0.5^{\circ}$. Despite the same motion, the joint torque (Figs. 3(a) and 3(b)) and tangential contact forces (Fig. 3(c)) show a largely different distribution. In fact, minimizing the torques (black) yields an overall energy consumption $E=\int \tau^{2} \mathrm{~d} t$ of $15 \%$ less than with the tangential force minimization (red). In return, the risk of slippage increases from $\bar{\mu}=\operatorname{mean}\left(\frac{F_{\text {tan }}}{F_{\text {norm }}}\right)=0.046$ to $\bar{\mu}=0.2$.

\section{B. Dynamic Trotting}

For dynamic trotting gaits (Fig. 2(b)), the robot continuously alternates the diagonal pairs of supporting legs.

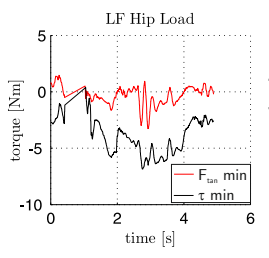

(a) Hip torque

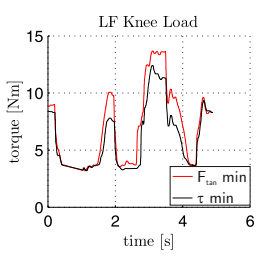

(b) Knee torque

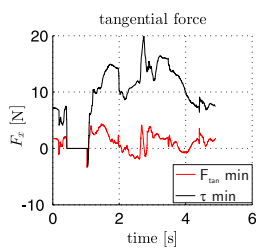

(c) Tangential force
Fig. 3. Efficiency (black) or risk of slippage (red) optimization results in different joint torque $(a, b)$ and contact force (c) distributions while the motion remains equal.

Thereby, the robot is statically unstable, as the angular momentum around the line of support cannot be changed (blue arrow). To control respectively stabilize this gait, we use the following tasks with decreasing priority: system dynamics (1), contact constraint (2), body orientation, body height, horizontal body velocity, and contact force alignment. Due to the system underactuation, the horizontal body velocity task is only fulfilled as well as possible in a LS sense. However, the gait can still be stabilized even under significant external disturbances by appropriately choosing the stepping location [34].

This trotting gait was successfully tested with different gait parameters like duty cycle or vertical oscillation of the main body but with the same control structure and gains. The picture series in Fig. 4 illustrates a trotting example on the treadmill with a speed of about $0.5 \mathrm{~m} / \mathrm{s}$. Although robustness against disturbances is hard to quantify, it was experimentally validated by external pulls and pushes or unperceived ground obstacles.

\section{Pronking while Exploiting Passive Dynamics}

Pronking is a dynamic gait with all four legs in synchronized gait phase and a substantial flight phase (Fig. 2(c)). In the present experiment, the robot achieves a jumping height (vertical distance between highest point in flight curve and height at lift-off) of about $0.11 \mathrm{~m}$ at a duty factor (relative time in ground contact) of about 0.5 (Fig. 5). In contrast to the two previous gaits, the main body is not kept at constant height but undergoes significant oscillations in vertical direction. Hence, to make such a gait energetically efficient, it is important to optimally excite the natural spring dynamics in the SEA joints which mostly contribute the vertical oscillation dynamics. Similarly to the early research by Raibert [35] or single legged hopping [20], the knee joints are solely used as thrust source by extending the knee motor with constant speed directly after landing. In the first phase after touchdown, the joint springs substantially deflect and hence store kinetic energy from the downward motion in potential spring energy. In the second phase after mid-stance, the entire body is accelerated upwards until liftoff. The task decomposition for the controller was chosen as follows with decreasing priority: system dynamics (1), contact constraint (2), passive dynamics in the knee joint (21), body orientation, horizontal body velocity, and contact force alignment. Importantly, the body height is not actively 


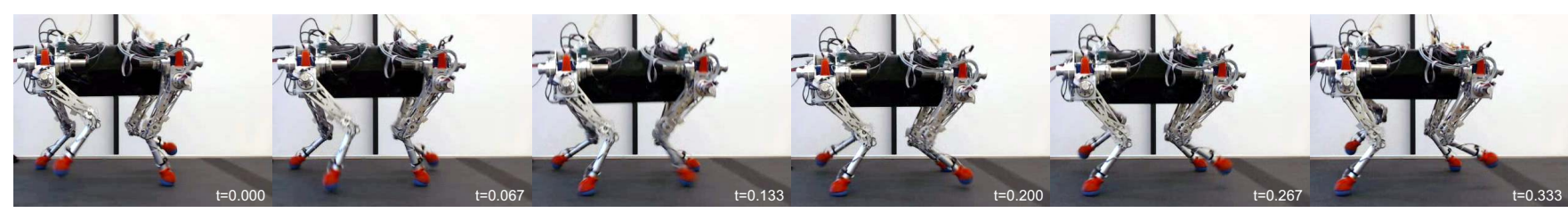

Fig. 4. Trotting on the treadmill with a locomotion speed about $0.5 \mathrm{~m} / \mathrm{s}$ or 1 bodylenght/s.

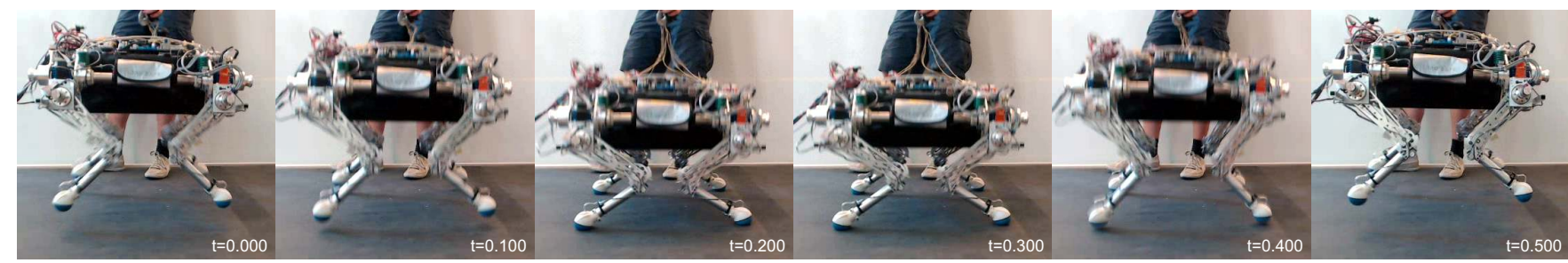

Fig. 5. A full gait cycle of pronking with a height of $0.11 \mathrm{~m}$ and a duty factor of about 0.56 .

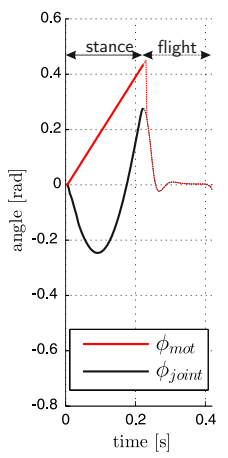

(a) simulation

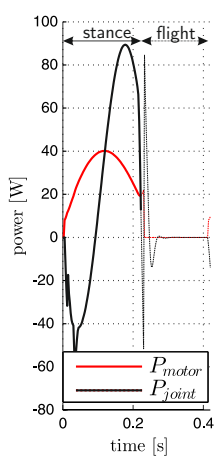

(d) simulation

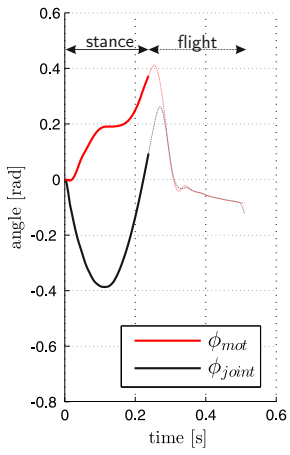

(b) passive dynamics

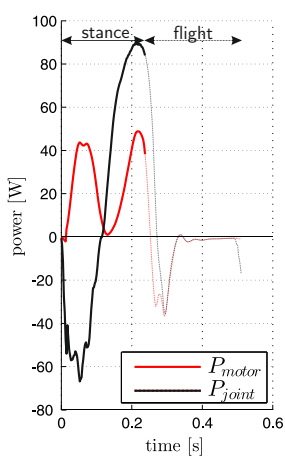

(e) passive dynamics

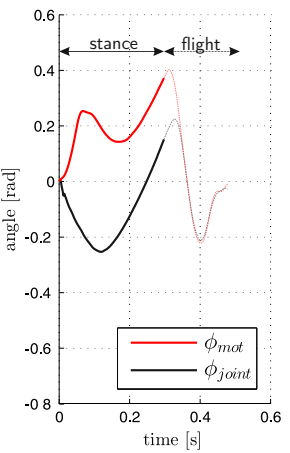

(c) pure feedback

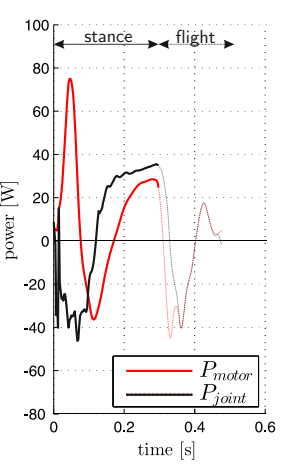

(f) pure feedback

Fig. 6. Position and power courses in the left front knee joint during pronking simulation $(\mathrm{a}, \mathrm{d})$ and experiments exploiting the passive dynamics $(\mathrm{b}, \mathrm{e})$ and with pure feedback control $(\mathrm{c}, \mathrm{f})$. By exploiting the passive dynamics $(\mathrm{a}, \mathrm{c})$ the motor travel distance, motor energy, and peak power can be reduced while the peak power at the joint is increased.

regulated as the vertical oscillatory motion evolves from the passive knee joint dynamics.

Figure 6 unveils some interesting findings when comparing the presented algorithm used to excite passive dynamics in simulation (Figs. 6(a) and 6(d)) and experiment (Figs. 6(b) and 6(e)) with a classical control approach where the motion is first optimized in simulation and then executed with pure feedback control on the system (Figs. 6(c) and 6(f), [18]). In the idealized simulation (Fig. 6(a)), the motor (red) expands with constant velocity while the knee (black) undergoes a

large motion with a deceleration phase until mid-stance and a slightly longer acceleration phase before lift-off. Expansion with constant velocity is not possible in the real experiments (Fig. 6(b)) due to saturation effects during mid-stance phase, which leads to a motor deceleration and a longer contact time with larger joint deflections. The classical control approach of following a desired main body trajectory (optimized in simulation) using pure feedback control (Fig. 6(b)) yields an even longer stance duration, whereby the motor velocity gets negative during mid-stance phase.

Active excitation of the passive spring dynamics has a beneficial impact on the power curve as the motor in Fig. 6(e) produces exclusively positive power throughout the entire stance phase while the joint first decelerates (energy is stored in the spring) and then re-accelerates again. In fact, the relation between the positive power the motor produces and the positive power the joint actually needs to maintain the pronking gait is only about $58 \%$. The rest is passively stored and released by the series elastic actuators. This is different for the pure feedback controller (Fig. 6(f)). The motor actively breaks and produces almost the same amount of energy as required at the joint (98\%). The second benefit can be seen in the peak power of both methods. When exploiting the passive dynamics, the motor peak power is below $50 \mathrm{~W}$, while the closed loop controller requires almost 80 W. Moreover, we can identify in (Fig. 6(e)) a clear peak power amplification from the motor to the joint by more than a factor 2 .

\section{Conclusion}

The contribution of this paper is two-pronged. First, we presented a hierarchical OSC method based on prioritized least squares optimization. Taking use of the special structure of the equations of motion, all tasks are expressed with a reduced optimization vector. Such control problem can be solved by iterative null-space projection or a numerical QP solver. The functionality and applicability of this approach was successfully evaluated in static walking and dynamic trotting experiments using the quadrupedal robot StarlETH [17]. Additionally to robust locomotion, it was demonstrated 
how the energetic efficiency and risk against slippage can be improved by optimally shaping the internal contact forces without changing the overall motion.

Second, we outlined a simple method that allows to excite the passive dynamics of distinct actuators while the motion is stabilized by hierarchical OSC. We employed this strategy in a pronking gait with a substantial flying phase. The generated excitation and motion shows major advantages in comparison to a traditional feedback control approach. The energy recuperation can be improved such that $42 \%$ of the energy turnover during stance is passively recovered by the series elasticity in the joints. In addition, the peak power at the motor is reduced by about $40 \%$ and the peak power at the joint is almost tripled. Astonishingly, large coincidence can be found when comparing the resulting power and position curves during stance phase with the observation in the muscular-tendon system of humans and animals during steady state running [36].

Going beyond the presented results, it is our goal to apply this hierarchical OSC controller to further gaits and combine it with the optimization framework presented in [18]. With this, we expect to excite and stabilize locomotion patterns that are impossible to achieve with pure feedback or pure open-loop control.

\section{REFERENCES}

[1] G. Hirzinger, A. Albu-Schaffer, M. Hahnle, I. Schaefer, and N. Sporer, "On a new generation of torque controlled light-weight robots," in International Conference on Robotics and Automation (ICRA), vol. 4, 2001, pp. 3356-3363.

[2] P. G. Gonzales de Santos, E. Garcia, and J. Estremera, "Stability in Walking Robots," in Quadrupedal Locomotion. An Introduction to the Control of Four-legged Robots. Springer, 2006, vol. 1, pp. 33-54.

[3] C. Samson, M. L. Borgne, and B. Espiau, Robot Control: The Task Function Approach. Oxford, U.K.: Clarendon Press, 1991.

[4] H. Hanafusa, T. Yoshikawa, and Y. Nakamura, "Analysis and control of articulated robot arms with redundancy," in Preprints 8th Triennial IFA C World Congress, vol. 14, Kyoto, 1981, pp. 78-83.

[5] B. Siciliano and J. J. E. Slotine, "A General Framework for Managing Multiple Tasks in Highly Redundant Robotic Systems," in International Conference on Robots in Unstructured Environments (ICAR), 1991, pp. 1211-1216 vol.2.

[6] O. Khatib, "A unified approach for motion and force control of robot manipulators: The operational space formulation," IEEE Journal of Robotics and Automation, vol. 3, no. 1, pp. 43-53, 1987.

[7] L. Sentis and O. Khatib, "Compliant Control of Multicontact and Center-of-Mass Behaviors in Humanoid Robots," IEEE Transactions on Robotics, vol. 26, no. 3, pp. 483-501, June 2010.

[8] R. Philippsen, L. Sentis, O. Khatib, and L. Sentist, "An open source extensible software package to create whole-body compliant skills in personal mobile manipulators," in IEEE/RSJ International Conference on Intelligent Robots and Systems (IROS), 2011, pp. 1036-1041.

[9] M. de Lasa and A. Hertzmann, "Prioritized optimization for task-space control," IEEE/RSJ Intenational Conference on Intelligent Robots and Systems (IROS), pp. 5755-5762, Oct. 2009.

[10] A. Escande, N. Mansard, and P.-B. Wieber, "Fast resolution of hierarchized inverse kinematics with inequality constraints," in IEEE International Conference on Robotics and Automation (ICRA). IEEE, May 2010, pp. 3733-3738.

[11] A. k. Björck, Numerical Methods for Least Squares Problems. Society for Industrial and Applied Mathematics, Jan. 1996.

[12] N. Mansard, "A dedicated solver for fast operational-space inverse dynamics," in IEEE International Conference on Robotics and Automation (ICRA). IEEE, May 2012, pp. 4943-4949.

[13] L. Righetti and S. Schaal, "Quadratic programming for inverse dynamics with optimal distribution of contact forces," in IEEE-RAS International Conference on Humanoid Robots, 2012, pp. 538-543.
[14] L. Saab, O. E. Ramos, F. Keith, N. Mansard, P. Soueres, and J.-Y. Fourquet, "Dynamic Whole-Body Motion Generation Under Rigid Contacts and Other Unilateral Constraints," IEEE Transactions on Robotics, vol. 29, no. 2, pp. 346-362, Apr. 2013.

[15] N. Mansard, O. Khatib, and A. Kheddar, "A Unified Approach to Integrate Unilateral Constraints in the Stack of Tasks," IEEE Transactions on Robotics, vol. 25, no. 3, pp. 670-685, June 2009.

[16] O. Kanoun, F. Lamiraux, and P.-B. Wieber, "Kinematic Control of Redundant Manipulators: Generalizing the Task-Priority Framework to Inequality Task," IEEE Transactions on Robotics, vol. 27, no. 4, pp. 785-792, Aug. 2011.

[17] M. Hutter, C. Gehring, M. Bloesch, M. Hoepflinger, C. D. Remy, and R. Siegwart, "StarlETH: a Compliant Quadrupedal Robot for Fast, Efficient, and Versatile Locomotion," in International Conference on Climbing and Walking Robots (CLAWAR), 2012, pp. 483-490.

[18] C. Gehring, S. Coros, M. Hutter, M. Bloesch, P. Fankhauser, M. A. Hoepflinger, and R. Siegwart, "Towards Automatic Discovery of Agile Gaits for Quadrupedal Robots," in IEEE International Conference on Robotics and Automation (ICRA), 2014.

[19] G. A. Pratt and M. M. Williamson, "Series elastic actuators," in IEEE International Conference on Intelligent Robots and Systems (IROS). Pittsburg, PA: MIT, 1995, Master Thesis, pp. 399-406.

[20] M. Hutter, C. D. Remy, M. H. Hoepflinger, and R. Siegwart, "High Compliant Series Elastic Actuation for the Robotic Leg ScarlETH," in International Conference on Climbing and Walking Robots (CLAWAR), Paris, Fr, 2011, pp. 507-514.

[21] I. Poulakakis, "On the Passive Dynamics of Quadrupedal Running," Ph.D. dissertation, Montreal, Canada, 2002.

[22] A. Sprowitz, A. Tuleu, M. Vespignani, M. Ajallooeian, E. Badri, and A. J. Ijspeert, "Towards dynamic trot gait locomotion: Design, control, and experiments with Cheetah-cub, a compliant quadruped robot," The International Journal of Robotics Research, vol. 32, no. 8, pp. 932-950, June 2013.

[23] R. M. Alexander, Elastic mechanisms in animal movement. Cambridge, Mass.: Cambridge University Press, 1988.

[24] L. Sentis and O. Khatib, "Control of Free-Floating Humanoid Robots Through Task Prioritization," in IEEE International Conference on Robotics and Automation (ICRA), 2005, pp. 1718-1723.

[25] L. Sentis, "Synthesis and Control of Whole-Body Behaviors in Humanoid Systems," Ph.D. dissertation, Stanford University, 2007.

[26] M. Mistry, J. Buchli, and S. Schaal, "Inverse Dynamics Control of Floating Base Systems Using Orthogonal Decomposition," in International Conference on Robotics and Automation (ICRA), 2010, pp. 3406-3412.

[27] F. Aghili, "A unified approach for inverse and direct dynamics of constrained multibody systems based on linear projection operator: applications to control and simulation," IEEE Transactions on Robotics, vol. 21, no. 5, pp. 834-849, 2005.

[28] L. Righetti, J. Buchli, M. Mistry, and S. Schaal, "Inverse dynamics control of floating-base robots with external constraints: A unified view," in International Conference on Robotics and Automation (ICRA), 2011, pp. 1085-1090.

[29] A. Herzog, L. Righetti, F. Grimminger, P. Pastor, and S. Schaal, "Momentum-based Balance Control for Torque-controlled Humanoids," Online Available, Tech. Rep., 2013.

[30] M. Hutter, M. Hoepflinger, C. D. Remy, and R. Siegwart, "Hybrid Operational Space Control for Compliant Legged Systems," in Robotics Science and Systems (RSS), 2012, pp. 129-136.

[31] M. Hutter, "StarlETH \& Co - Design and Control of Legged Robots with Compliant Actuation," Ph.D. dissertation, ETH Zurich, 2013.

[32] A. Weinmann, Uncertain Models and Robust Control. Springer, 1991.

[33] C. D. Remy, K. W. Buffinton, and R. Siegwart, "Comparison of Cost Functions for Electrically Driven Running Robots," in IEEE International Conference on Robotics and Automation (ICRA). IEEE, May 2012, pp. 2343-2350.

[34] C. Gehring, S. Coros, M. Hutter, M. Bloesch, M. A. Hoepflinger, and R. Siegwart, "Control of dynamic gaits for a quadrupedal robot," in IEEE International Conference on Robotics and Automation (ICRA). Karlsruhe, Germany: IEEE, May 2013, pp. 3287-3292.

[35] M. H. Raibert, Legged robots that balance. Cambridge, Mass.: MIT Press, 1986.

[36] T. J. Roberts, "The integrated function of muscles and tendons during locomotion." Comparative biochemistry and physiology. Part A, Molecular \& integrative physiology, vol. 133, no. 4, pp. 1087-99, Dec. 2002. 